\title{
Induced gravity and gauge interactions revisited
}

\author{
Bogusław Broda*, Michał Szanecki \\ Department of Theoretical Physics, University of Łódź, Pomorska 149/153, 90-236 Łódź, Poland
}

\section{A R T I C L E I N F O}

\section{Article history:}

Received 14 January 2009

Received in revised form 17 February 2009

Accepted 27 February 2009

Available online 5 March 2009

Editor: T. Yanagida

\section{PACS:}

11.15.Tk

$04.62 .+\mathrm{v}$

04.70.Dy

12.10.Dm

\begin{abstract}
A B S T R A C T
It has been shown that the primary, old-fashioned idea of Sakharov's induced gravity and gauge interactions, in the "one-loop dominance" version, works astonishingly well yielding phenomenologically reasonable results. As a byproduct, the issue of the role of the UV cutoff in the context of the induced gravity has been reexamined (an idea of self-cutoff induced gravity). As an additional check, the black hole entropy has been used in the place of the action. Finally, it has been explicitly shown that the induced coupling constants of gauge interactions of the standard model assume qualitatively realistic values.
\end{abstract}

(C) 2009 Elsevier B.V. All rights reserved.

\section{Introduction}

The idea that fundamental interactions might be not so fundamental as they appear, but induced by quantum fluctuations of the vacuum emerges from the fifties of the 20th century. In 1967 Sakharov published his famous short paper on induced gravity [1], and in the same year Zel'dovich presented a parallel result concerning electrodynamics [2]. The both authors have made use of some earlier observations coming from papers (cited by them) by Landau and his collaborators. In fact, the induced gauge theory was initiated four years earlier in [3], and next followed by many people (see, e.g. [4]). It was applied to the standard model in [5], whereas field theoretical realizations and calculations concerning induced gravity were given in [6,7]. A logarithmic relation between the gauge coupling constants and the Newton gravitational constant has been noticed in [8] (this relation has been derived in [9] using another requirement). Some further, related aspects have been elaborated in $[10,11]$. It seems that successful application of the idea of quantum vacuum induced interactions to the two fundamental interactions subsequently renewed interest in this subject. Actually, at present, the very idea lacks a clear theoretical interpretation. It can be treated either as an interesting curiosity or as an unexplained deeper phenomenon. Anyway, coincidences are striking. Our point of view is purely pragmatical, i.e. we claim that the idea of quantum induced interactions does work.

\footnotetext{
* Corresponding author.

E-mail addresses: bobroda@uni.lodz.pl (B. Broda), michalszanecki@wp.pl (M. Szanecki).
}

The aim of our Letter is to show that the idea of induced gauge interactions (including gravity and, possibly, dark energy) in its primary, old-fashioned, Sakharov's version yields phenomenologically very realistic results. The phrase "primary, old-fashioned, Sakharov's version" means "one-loop dominance" interpretation in the terminology of a review paper on Sakharov's induced gravity [12]. This standpoint assumes that at the beginning there are no classical terms for gauge fields and gravity. There are only (fundamental) matter fields present in the classical action, and they are coupled to external gauge fields and gravity. (The superior role of the matter fields awaits an explanation in this framework.) Interestingly, and it was primary inspiration, it appears that low-order one-loop calculations yield proper classical terms for gauge and gravitational fields. Just only this fact, akin to renormalizability, is by no means surprising. But what is really surprising is that not only appropriate functional terms emerge from these one-loop matter field calculations but phenomenologically realistic numeric coefficients as well.

As far as a conceptual side of the idea is concerned, in the case of gravity, we have also proposed an alternative point of view. Actually, there is some logical gap in the standard approach which consists in imposing the Planck cutoff to derive the strength of gravitational interactions which subsequently yields the Planck cutoff itself. Namely, we propose to shift the focus to analyzing the role of relation between the "Schwarzschild" radius and the mass, leaving the Newton gravitational constant undetermined (self-cutoff induced gravity). We have also suggested to use the entropy instead of the action as an independent check of the whole procedure. Finally, we have explicitly estimated coupling constants of fundamental interactions, which appear to assume realistic values. In our Letter, gravity (possibly, including dark energy) and gauge interactions are treated uniformly, i.e. the both kinds of in- 
teractions are analysed parallelly and the both kinds of interactions are approached in the framework of the same and very convenient method: Schwinger's proper time and the Seeley-DeWitt heat-kernel expansion. One should mention that the natural idea of using the heat-kernel expansion (in the euclidean version) to get the gravitational induced action was introduced in [13], whereas temperature dependence of the induced coupling constants, and its potential impact on black hole evaporation, has been investigated in [14]. Finally an extension including torsion has been given in [15].

\section{Heat-kernel method}

According to the idea of quantum vacuum induced interactions, dynamics of gravity (possibly, including also dark energy) and gauge interactions emerges from dominant contributions to the one-loop effective action of non-self-interacting matter fields coupled to these interactions. In the framework of the Schwinger proper-time method, the expected terms for "cosmological constant" (dark energy), gravity and gauge interactions can be extracted from the 0th, 1st and 2nd coefficient of the Seeley-DeWitt heat-kernel expansion, respectively. In Minkowskian signature [16, 17]

$S_{\text {eff }}=i \kappa \log \operatorname{det} \mathcal{D}=i \kappa \operatorname{Tr} \log \mathcal{D}=-i \kappa \int \frac{\mathrm{d} s}{s} \operatorname{Tr} e^{-i s \mathcal{D}}$,

where $\mathcal{D}$ is an appropriate second-order differential operator, and $\kappa$ depends on the kind of the "matter" field (its statistics, in principle). E.g. for a scalar mode, $\kappa=\frac{1}{2}$. Making use of the SeeleyDeWitt heat-kernel expansion in four dimensions,

$\operatorname{Tr} e^{-i s \mathcal{D}}=\frac{1}{16 \pi^{2}(i s)^{2}}\left[A_{0}+A_{1}(i s)+A_{2}(i s)^{2}+\cdots\right]$,

where $A_{n}$ is the $n$th Seeley-DeWitt coefficient, and next imposing appropriate cutoffs, i.e. an UV cutoff $\varepsilon$ for $A_{0}, A_{1}$ and $A_{2}$, and an IR cutoff $\Lambda$ for $A_{2}$, we obtain

$S_{\text {eff }}=\frac{\kappa}{16 \pi^{2}}\left(\frac{1}{2} A_{0} \varepsilon^{-2}+A_{1} \varepsilon^{-1}+A_{2} \log \frac{\Lambda}{\varepsilon}+\cdots\right)$.

Collecting contributions from various modes, we get the following Lagrangian densities:

$\mathcal{L}_{0}=\frac{1}{64 \pi^{2}} \varepsilon^{-2}\left(N_{0}-2 N_{\frac{1}{2}}+2 N_{1}\right)$,

$\mathcal{L}_{1}=-\frac{1}{192 \pi^{2}} \varepsilon^{-1}\left(N_{0}+N_{\frac{1}{2}}-4 N_{1}\right) R$,

and

$\mathcal{L}_{2}=\frac{1}{384 \pi^{2}} \log \frac{\Lambda}{\varepsilon}\left(N_{0}+4 N_{\frac{1}{2}}\right) \operatorname{tr} F^{2}$

(see Table 2 in Appendix A for the origin of the numeric coefficients), where:

$N_{0}=$ number of minimal scalar degrees of freedom (dof),

$N_{\frac{1}{2}}=$ number of two-component fermion fields

$=$ half the number of fermion dof,

$N_{1}=$ number of gauge fields $=$ half the number of gauge dof.

The Lagrangian densities $\mathcal{L}_{0}, \mathcal{L}_{1}$ and $\mathcal{L}_{2}$ correspond to the terms $A_{0}, A_{1}$ and $A_{2}$ in (3), and yield the cosmological constant, Einstein's gravity and gauge interactions, respectively. (Here $R$ is the scalar curvature, and $F$ is the strength of a gauge field, see, the definition (A.1).) Higher-order terms are in principle present (even in classical case), but they are harmless in typical situations because of small values of the coefficients following from the cutoffs. An exception appears and is discussed in Section 3.2.
The infamous cosmological constant directly follows from Eq. (4) but it is unacceptable in this form because its value is too huge [18], i.e. it is at least $10^{120}$ times greater than expected. Therefore, $A_{0}$ could, in principle, spoil the idea of induced interactions but it is not necessarily so. It appears [19] that it is, in principle, possible to tame the expression (4), so preserving the concept of induced interactions consistent.

In the following sections we will consider induced gravity and standard model gauge interactions.

\section{Induced gravity}

\subsection{Standard approach}

Assuming the commonly being used, standard, Planckian value of the UV cutoff, $\varepsilon=G$ (= the Newton gravitational constant), we directly obtain from (5)

$\mathcal{L}_{1}=-\frac{1}{12 \pi}\left(N_{0}+N_{\frac{1}{2}}-4 N_{1}\right) \frac{1}{16 \pi G} R$.

Intuitively, the (quantum) Planckian cutoff can be explained as following from a classical gravitational cutoff imposed by the black hole horizon. Namely, the description of matter in terms of particles, or even the notion of particles itself, is not valid for particles of enormous, i.e. Planckian, masses because of the mechanism of black-hole formation (see, Fig. 1). We shall return to this thread in the next subsection.

The result (8) has been already explicitly presented in [20] (there is a misprint in the coefficient in front of $N_{1}$ of his final formula (4.2)). It has been also rederived in the framework of the heat-kernel method, in the context of supersymmetry, in [21]. Finally, Eq. (8) can also be easily recovered from the data given in [12]. The aim of the former two papers was to investigate the influence of gauge fields on the sign of $\mathcal{L}_{1}$. Obviously, the gauge fields tend to change the sign of $\mathcal{L}_{1}$. Instead, our aim, in this subsection, is to emphasize that Eq. (8) directly yields a realistic value of the Newton gravitational constant, provided the Planckian UV cutoff is given. Namely, putting, e.g., $N_{0}=0, N_{\frac{1}{2}}=45$ and $N_{1}=0$ in (8) we get in front of the standard Hilbert-Einstein action $\frac{45}{12 \pi} \approx 1.19=\mathcal{O}(1)$, which is an impressive coincidence. Taking into account an approximate character of the derivation such a high precision is absolutely unnecessary and seems to be rather accidental. Therefore, assuming non-zero $N_{0}$ and $N_{1}$, e.g. $N_{0}=4$ and $N_{1}=12$, yields $\frac{1}{12 \pi} \approx 0.03$, and it is perhaps less impressive but phenomenologically acceptable as well. The proposed value of $N_{\frac{1}{2}}$ corresponds to $3 \times(3+3 \times 4)=45$ fermion two-component field species contained in the standard model ( 3 families of leptons and quarks in 3 colors). In the second example, we admit the existence of the Higgs scalar, $N_{0}=4$, and the contribution of $N_{1}=1+3+8=12$ gauge fields. Since the gauge fields themselves are also induced entities, their contribution to the count is disputable. The existence of the Higgs particle itself is disputable as well.

\subsection{Alternative (cutoff independent or self-cutoff) approach}

Strictly speaking, the whole approach presented in the previous subsection, and being in accordance with a standard, commonly accepted point of view, is not quite logically consistent. The lack of the full logical consistency is a consequence of the fact that the assumed Planckian value of the UV cutoff, in principle, follows from the value of the (effective) Newton gravitational constant that is just being induced. In other words, a consistent reasoning should be independent of any explicit value of the UV cutoff. Of course, it is impossible to derive the (numeric) value of $G$ or, equivalently, of 
the UV cutoff in such a framework, but nevertheless some physically non-trivial conclusions can be drawn.

First of all, we should somehow explain the appearance of the Planck scale in physics. Apparently, there are the two main points of view in this respect. The first point of view, due to Planck himself, appeals to a possibility to construct an appropriate dimensionfull quantity out of several fundamental physical constants. It is theory independent and natural for simple dimensional grounds, but it lacks a firm physical support. Besides, that approach is not able to yield any purely numeric coefficient. The second approach instead tries to derive the Planck scale using some physical, theory grounded arguments. That approach dates back to the papers [22-24], and conforms to our point of view. Roughly, the idea, one could call self-cutoff induced gravity, consists in identification of the Schwarzschild diameter and the Compton wavelength. Naively, the reasoning according to these guidelines could look like follows. Literally repeating the derivation of (8), but this time with an unpredefined UV cutoff $M$ (in mass units), we get

$\mathcal{L}_{1}=-\frac{M^{2}}{16 \pi} \cdot \frac{N}{12 \pi} R$,

where $N$ is the "effective number" of particle species, e.g. $N=$ $N_{0}+N_{\frac{1}{2}}-4 N_{1}$ (see (8)). Obviously, the Schwarzschild solution of the Einstein equation following from (9) is independent of the coefficients in (9), and it assumes the well-known form

$d s^{2}=\left(1-\frac{\mu}{r}\right) d t^{2}-\left(1-\frac{\mu}{r}\right)^{-1} d r^{2}-r^{2} d \Omega^{2}$,

where $\mu$ is an as yet undefined parameter. The (standard) linearized and well-known version of the Einstein equation for the metric (10) (Newtonian limit) with a point-like source representing a particle of the highest admissible mass $M$ (UV cutoff) with coefficients of Eq. (9) reads

$\frac{M^{2}}{16 \pi} \cdot \frac{N}{12 \pi} \cdot \frac{1}{2} \Delta\left(-\frac{2 \mu}{r}\right)=\frac{1}{2} M \delta^{3}(\mathbf{r})$,

where $\Delta$ is the three-dimensional Laplacian, and $\delta^{3}(\mathbf{r})$ is the Dirac delta. From (11), it follows that

$M N \mu=24 \pi$.

On the other hand, the value of the Compton wavelength for the particle of the mass $M$ is

$\lambda_{c}=\frac{2 \pi}{M}$.

Equating $\lambda_{c}$ and the Schwarzschild diameter

$2 r_{s}=2 \mu$,

we obtain from (12) the final (dimensionless) result:

$N=24$.

Typically $r_{s} \ll \lambda_{c}$ and therefore no gravity concepts enter quantum theory discussion. But when the mass $M$ grows, $r_{s}$ grows linearly, whereas $\lambda_{c}$ decreases. When the both values become comparable the particle can be intuitively considered as trapped in its own black hole (Fig. 1).

We would like to emphasize that the constraint (14) which is qualitatively fully consistent with our earlier ones (derived in Section 3.1) is derived owing to the self-cutoff assumption but not in general induced gravity. In this place, we could hastily conclude that the focus is now shifted to analyzing the role of the "effective number" of degrees of freedom of matter fields (see Eq. (14)). Unfortunately, such a conclusion would be not quite correct because there is a problem with higher-order Seeley-DeWitt coefficients. They are harmless for small Riemann curvature $R$, but when the

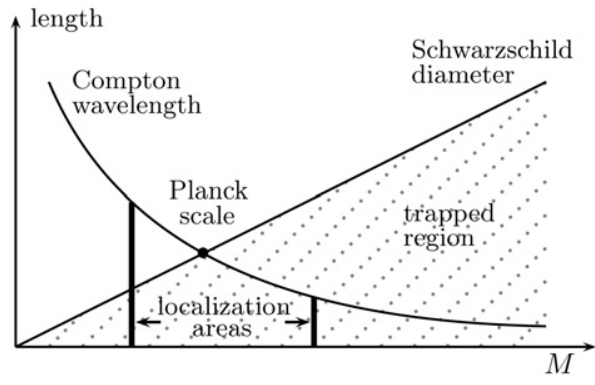

Fig. 1. Qualitative picture of the emergence of the Planck scale. The left localization area corresponds to the regime of standard particle-field theory formalism, whereas the right one is outside the scope of this formalism.

Riemann curvature $R$ is of the order of $M^{2}$, an infinite tower of $\left(R / M^{2}\right)^{n}$ corrections to the Einstein term appears ( $R$ denotes here not only the scalar curvature but symbolically all kinds of curvature terms of the corresponding dimension). This could, in principle, invalidate the whole argumentation yielding the result (14). Therefore, we claim that the proper conclusion to be drawn in the end of this section is as follows. The derivation is consistent, and the phenomenologically reasonable result $N \approx 24$ is obtained provided the formula for the Schwarzschild radius (13) is stable against the influences of the infinite tower of higher-curvature corrections.

Strictly speaking, we should expect some form of a generalization of the Schwarzschild solution and of the Schwarzschild radius. The well-known linear relation between the radius of the event horizon and the mass (13) follows from the form of the Schwarzschild solution of the Einstein equation according to the argumentation presented between Eq. (9) and Eq. (11). It is true for $\left|R_{H}\right| \ll M^{2}$ (the curvature $R$ at the event horizon $H$ is much less than the UV cutoff), otherwise the Hilbert-Einstein action is modified by higher-order terms in $R$, and the classical result could be invalidated. But it appears that phenomenologically the "effective number" of particle species $N$ qualitatively conforms to (13). This result confirms that the derivation is somehow insensitive to terms of higher-order in $R$. Such a kind of the result could be of interest in application to mini black holes where, in principle, one should not discard higher-order terms.

\subsection{Entropy}

In this subsection, we would like to draw reader's attention to an independent argument in favour of the idea of induced gravity. Namely, we will show that not only the action but also the entropy sums up appropriately, i.e. gravitational entropy of a black hole can be recovered from entropies of "fundamental" fields. In other words, what applies to the actions remains in force in the case of the entropies. A slight complication follows from the fact that the notion of the entropy is not quite unique. In principle, in the context of gravity usually two notions of the entropy appear: so-called, "geometrical entropy" and "thermodynamical entropy". Since the derivation of the geometrical entropy [25] uses the heat-kernel method, the result for the entropy is analogous to the previous one for the action. Namely, in close analogy to Eq. (8) we have

$S_{g}=\frac{1}{12 \pi}\left(N_{0}+N_{\frac{1}{2}}-4 N_{1}\right) S_{A}$,

where $S_{g}$ denotes the geometrical entropy and $N_{0}, N_{\frac{1}{2}}, N_{1}$ are defined in (7). Here, the black hole entropy

$S_{A}=\frac{1}{4 G} A$, 
Table 1

Contributions to induced gauge field coupling constants coming from various "matter fields" species in the framework of the standard model.

\begin{tabular}{|c|c|c|c|}
\hline gauge group & $\mathrm{U}(1)$ & $\mathrm{SU}(2)$ & $\mathrm{SU}(3)$ \\
\hline coupling constant & $g^{\prime}$ & $g$ & $f$ \\
\hline "coupling matrix" & $\frac{i}{2} Y g^{\prime}$ & $\frac{i}{2} \vec{\tau} g$ & $\frac{i}{2} \vec{\lambda} f$ \\
\hline conventions & $Y$-hypercharge & $\operatorname{tr}\left(\tau^{a} \tau^{b}\right)=2 \delta^{a b}, a, b=1,2$ & $\operatorname{tr}\left(\lambda^{i} \lambda^{j}\right)=2 \delta^{i j}, i, j=1,2,3$ \\
\hline \multicolumn{4}{|c|}{ FERMIONS (one family) $a_{2}=-\frac{1}{3} \operatorname{tr} F^{2}$} \\
\hline numeric formula & $-\frac{1}{3}\left(-\frac{1}{4}\right) Y^{2} g^{\prime 2}=\frac{1}{12} Y^{2} g^{\prime 2}$ & $-\frac{1}{3} \cdot 2\left(-\frac{1}{4}\right) g^{2}=\frac{1}{6} g^{2}$ & $-\frac{1}{3} \cdot 2\left(-\frac{1}{4}\right) f^{2}=\frac{1}{6} f^{2}$ \\
\hline \multicolumn{4}{|l|}{ LEPTONS } \\
\hline left, $Y=-1$ & $\frac{1}{12} \cdot 2 g^{\prime 2}=\frac{1}{6} g^{\prime 2}$ & $\frac{1}{6} g^{2}$ & 0 \\
\hline right, $Y=-2$ & $\frac{1}{12} \cdot(-2)^{2} g^{\prime 2}=\frac{1}{3} g^{\prime 2}$ & 0 & 0 \\
\hline \multicolumn{4}{|l|}{ QUARKS } \\
\hline left, $Y=\frac{1}{3}$ & $\frac{1}{12} \cdot 2 \cdot 3\left(\frac{1}{3}\right)^{2} g^{\prime 2}=\frac{1}{18} g^{\prime 2}$ & $\frac{1}{6} \cdot 3 g^{2}=\frac{1}{2} g^{2}$ & $\frac{1}{6} \cdot 2 f^{2}=\frac{1}{3} f^{2}$ \\
\hline right, $Y=\frac{4}{3}$ & $\frac{1}{12} \cdot 3\left(\frac{4}{3}\right)^{2} g^{\prime 2}=\frac{4}{9} g^{\prime 2}$ & & $\frac{1}{6} f^{2}$ \\
\hline right, $Y=-\frac{2}{3}$ & $\frac{1}{12} \cdot 3\left(-\frac{2}{3}\right)^{2} g^{\prime 2}=\frac{1}{9} g^{\prime 2}$ & 0 & $\frac{1}{6} f^{2}$ \\
\hline \multicolumn{4}{|c|}{ BOSONS $a_{2}=-\frac{1}{12} \operatorname{tr} F^{2}$} \\
\hline Higgs, $Y=1$ & $-\frac{1}{12} \cdot 2\left(-\frac{1}{4}\right) g^{\prime 2}=\frac{1}{24} g^{\prime 2}$ & $-\frac{1}{12} \cdot 2\left(-\frac{1}{4}\right) g^{2}=\frac{1}{24} g^{2}$ & 0 \\
\hline
\end{tabular}

where $A$ is the area of the black hole horizon. Actually, we reproduce the same bound as that given in (14), provided the expected value of $S_{g}=S_{A}$.

It appears that the approach making use of the thermodynamical entropy yields a slightly other result. Now, we have [26]

$S_{B}=\frac{1}{90 \pi} S_{A}$,

and

$S_{F}=\frac{7}{16} \cdot \frac{1}{90 \pi} S_{A}$,

for a bosonic and a fermionic degree of freedom, respectively. Therefore, the final formula reads

$S_{t}=\frac{1}{90 \pi}\left(N_{B}+\frac{7}{16} N_{F}\right) S_{A}$,

where $N_{B}$ and $N_{F}$ is the number of bosonic and fermionic degrees of freedom, respectively. In terms of $N_{0}, N_{\frac{1}{2}}, N_{1}$ we could rewrite (19) as (see, Table 2 and further formulas in Appendix A)

$S_{t}=\frac{1}{90 \pi}\left(N_{0}+\frac{7}{8} N_{\frac{1}{2}}+2 N_{1}\right) S_{A}$.

Evidently, the formula (20) differs from (15) but nevertheless qualitatively the result is essentially the same as earlier for the considered combinations of field species.

In the end of this subsection, we would like to stress that the presented observations are not new, only the point of view is changed. From traditional point of view, the most natural way to explain the origin of the black hole entropy is to treat it as "entanglement entropy" for constituent fields [27]. Accordingly, Eq. (15) is interpreted as a derivation of $S_{g}$. From our point of view the, so-called, "species problem", signaled in [27], does not exist.

\section{Induced gauge interactions}

In this section, we will concentrate on the possibility of quantum generation of gauge interactions in the context of the standard model. From technical point of view, we will be interested in the second Seeley-DeWitt coefficients for appropriate matter fields. The corresponding term has been already given in (6) but now we would like to adapt it to the context of the standard model. Adopting the matter contents of the Lagrangian of the standard model we display all contributions to the respective gauge parts in Table 1.

Here the assumed implicit convention for the operator of covariant derivative is

$D_{\mu}=\partial_{\mu}+\vec{X} \cdot \vec{A}_{\mu}$,

where $\vec{X}$ is the "coupling matrix" given in the third row of Table 1 . More precisely, $\vec{X}$ is a tensor product with two matrix units corresponding to the other two gauge groups, yielding additional coefficients, 2 or 3 . In principle, the coefficients given in each column and multiplied by

$\frac{1}{16 \pi^{2}} \log \frac{M}{m}$,

where $M$ and $m$ is an UV and an IR cutoff, respectively, in mass units, should sum up to $\frac{1}{4}$, a standard normalization term in front of $F^{2}$. More generally, we have the following theoretical bound:

$\frac{g_{i}^{2}}{16 \pi^{2}} \sum_{n} \alpha_{(i) n} \log \frac{M_{n}}{m_{n}}=\frac{1}{4}$,

where $g_{i}(i=1,2,3)$ is one of the three coupling constants, $\alpha_{(i) n}$ are corresponding numeric coefficients from Table 1 , and the sum concerns all matter fields. We can confidently set $M_{n}=M_{\mathrm{P}}$ (Planck mass), but the choice of $m_{n}$ is less obvious. Fortunately, the logarithm is not very sensitive to a change of the argument.

Now, the data given in Table 1 can be used to reproduce a number of phenomenologically realistic results. Assuming for simplicity (or as an approximation) fixed values of $M_{n}$ and $m_{n}$ for all species of matter particles, we can uniquely rederive following [28] the Weinberg angle $\theta_{\mathrm{w}}$,

$\sin ^{2} \theta_{\mathrm{w}}=\frac{g^{\prime 2}}{g^{2}+g^{\prime 2}} \approx 0.38$.

Unfortunately, estimation of the coupling constants requires definite values of infrared cutoffs $m_{n}$. Anyway, for $m_{n}$ of the order of the mass of lighter particles of the standard model we obtain

$\alpha=\frac{e^{2}}{4 \pi}=\frac{g^{2} \sin ^{2} \theta_{\mathrm{w}}}{4 \pi}=\mathcal{O}(0.01)$,

and

$g=f=\mathcal{O}(1)$,

which is phenomenologically a very realistic estimate. 
Table 2

Seeley-DeWitt coefficients and entropy coefficients. In brackets, we have given the references where the coefficients can be found explicitly or almost explicitly (i.e. after few-minute calculations).

\begin{tabular}{|c|c|c|c|c|}
\hline \multirow[t]{2}{*}{ Particle } & \multicolumn{3}{|c|}{ Seeley-DeWitt coefficients } & \multirow{2}{*}{$\begin{array}{l}\text { Entropy } \\
\text { coefficient } l\end{array}$} \\
\hline & $k_{0}$ & $k_{1}$ & $k_{2}$ & \\
\hline minimal scalar & 1 & $\frac{1}{6}[17]$ & $\frac{1}{12}[17]$ & $1[26]$ \\
\hline Weyl spinor & 2 & $-\frac{1}{6}[17]$ & $-\frac{1}{3}[17]$ & $\frac{7}{8}[26]$ \\
\hline massless vector & 2 & $-\frac{2}{3}[16]$ & $-\frac{11}{24}[29]$ & $2[26]$ \\
\hline
\end{tabular}

Alternatively, the bound (14) can give some, for example, (nonunique) limitations on the ratio of the two scales $M$ and $m$, provided the scale of interactions $g=f=\mathcal{O}(1)$ is assumed.

\section{Final remarks}

In this Letter, we have presented a number of arguments supporting the idea of the old-fashioned "one-loop dominance" version of induced gravity and gauge interactions in the spirit of Sakharov. All coupling constants of fundamental gauge interactions, including gravity, have been shown to assume phenomenologically realistic values, provided the Planckian value of the UV cutoff is given. Besides the action, also the black hole entropy has been shown to fit this picture. As another, UV cutoff free interpretation of the consistency of induced gravity, an estimate of the generalized Schwarzschild radius has been proposed in the framework of an idea of self-cutoff induced gravity.

It seems that the brane induced gravity could be probed with the approach presented. For example, in five dimensions, at least formally, we would have got $M_{*}^{3}$ instead of $M^{2}$ in front of the fivedimensional version of Eq. (9). In this case some difficulty could follow from the fact that the matter fields gravity is supposed to be induced from do not live in higher dimensions. But this subject is outside the scope of our Letter.

\section{Acknowledgements}

We are grateful to many persons for their critical and fruitful remarks which we have utilized in this Letter. This work was supported in part by the Polish Ministry of Science and Higher Education Grant PBZ/MIN/008/P03/2003 and by the University of Łódź grant.

\section{Appendix A. Seeley-DeWitt and entropy coefficients}

For reader's convenience we present below (in Table 2) the Seeley-DeWitt ("Hamidew") coefficients and the entropy coefficients used (except $k_{2}$ for a massless vector) in the main text. In the terminology of Misner, Thorn and Wheeler our sign convention corresponds to the Landau-Lifshitz timelike one, i.e. the metric signature is $(+---)$ and $R_{\beta \gamma \delta}^{\alpha}=\partial_{\gamma} \Gamma_{\beta \delta}^{\alpha}-\cdots$. Our conventions concerning gauge fields are as follows:

$D_{\mu}=\nabla_{\mu}+A_{\mu}$,

$F_{\mu \nu}=\partial_{\mu} A_{\nu}-\partial_{\nu} A_{\mu}+\left[A_{\mu}, A_{\nu}\right]$.

We have assumed the following notation:

$a_{0}(x)=k_{0}$,

$a_{1}(x)=-k_{1} R$

$a_{2}(x)=k_{2} \operatorname{tr} F^{2}+k_{2}^{\prime}$. "curvature terms",

and

$S_{t}=l \cdot \frac{1}{90 \pi} \cdot \frac{A}{4 G}$.

Interested reader can find $k_{2}^{\prime}$ in $[12,16,29]$.

\section{References}

[1] A.D. Sakharov, Gen. Relativ. Gravit. 32 (2000) 365, translated from Dokl. Akad. Nauk SSSR 170 (1967) 70.

[2] Y.B. Zel'dovich, Pis'ma Zh. Eksp. Teor. Fiz. 6 (1967) 922, JETP Lett. 6 (1967) 345

[3] J.D. Bjorken, Ann. Phys. 24 (1963) 174.

[4] I. Białynicki-Birula, Phys. Rev. 130 (1963) 465.

[5] H. Terazawa, Y. Chikashige, K. Akama, Phys. Rev. D 15 (1977) 480.

[6] K. Akama, Y. Chikashige, T. Matsuki, Prog. Theor. 59 (1978) 653; K. Akama, Y. Chikashige, T. Matsuki, H. Terazawa, Prog. Theor. Phys. 60 (1978) 868.

[7] K. Akama, Prog. Theor. Phys. 60 (1978) 1900.

[8] H. Terazawa, Y. Chikashige, K. Akama, T. Matsuki, Phys. Rev. D 15 (1977) 1181.

[9] L.D. Landau, On the quantum theory of fields, in: W. Pauli (Ed.), Niels Bohr and the Development of Physics (Essays dedicated to Niels Bohr on the occasion of his seventieth birthday), McGraw-Hill, New York, 1955, pp. 52-69.

[10] K. Akama, Pregeometry, in: K. Kikkawa, N. Nakanishi, H. Nariai (Eds.), Gauge Theory and Gravitation, Proceedings, Nara, 1982, Lecture Notes in Physics, vol. 176, Springer-Verlag, 1983, pp. 267-271, hep-th/0001113.

[11] K. Akama, Phys. Rev. Lett. 76 (1996) 184; K. Akama, T. Hattori, Phys. Rev. Lett. 93 (2004) 211602.

[12] M. Visser, Mod. Phys. Lett. A 17 (2002) 977, gr-qc/0204062.

[13] G. Denardo, E. Spallucci, Nuovo Cimento A 69 (1982) 151.

[14] G. Denardo, E. Spallucci, Phys. Lett. B 130 (1983) 43; G. Denardo, E. Spallucci, Nuovo Cimento A 74 (1983) 450.

[15] G. Denardo, E. Spallucci, Class. Quantum Grav. 4 (1987) 89.

[16] N.D. Birrell, P.C.W. Davies, Quantum Fields in Curved Space, Cambridge Univ. Press, 1982, Chapter 6.

[17] B. DeWitt, The Global Approach to Quantum Field Theory, Oxford Science Publications, 2003, Chapter 27.

[18] S. Weinberg, Rev. Mod. Phys. 61 (1989) 1.

[19] B. Broda, P. Bronowski, M. Ostrowski, M. Szanecki, Ann. Phys. (Berlin) 12 (11) (2008) 855, arXiv:0708.0530.

[20] K. Akama, Phys. Rev. D 24 (1981) 3073.

[21] M. Tanaka, Phys. Rev. D 53 (1996) 6941, hep-ph/9504259.

[22] A. Peres, N. Rosen, Phys. Rev. 118 (1960) 335.

[23] C.A. Mead, Phys. Rev. 135 (1964) B849.

[24] C.A. Mead, Phys. Rev. 143 (1966) 990.

[25] F. Larsen, F. Wilczek, Nucl. Phys. B 458 (1996) 249, hep-th/9506066.

[26] L. Zhong-heng, Int. J. Theor. Phys. 39 (2000) 253.

[27] T. Jacobson, gr-qc/9404039.

[28] H. Terazawa, K. Akama, Y. Chikashige, Prog. Theor. Phys. 56 (1976) 1935.

[29] D.V. Vassilevich, Phys. Rep. 388 (2003) 279, hep-th/0306138. 